\title{
IDENTITIES OF THE LEFT-SYMMETRIC WITT ALGEBRAS
}

\author{
Daniyar Kozybaev \\ and \\ Ualbai Umirbaev?
}

\begin{abstract}
Let $P_{n}=k\left[x_{1}, x_{2}, \ldots, x_{n}\right]$ be the polynomial algebra over a field $k$ of characteristic zero in the variables $x_{1}, x_{2}, \ldots, x_{n}$ and $\mathscr{L}_{n}$ be the left-symmetric Witt algebra of all derivations of $P_{n}$ [2]. We describe all right operator identities of $\mathscr{L}_{n}$ and prove that the set of all algebras $\mathscr{L}_{n}$, where $n \geq 1$, generates the variety of all left-symmetric algebras. We also describe a class of general (not only right operator) identities for $\mathscr{L}_{n}$.
\end{abstract}

Mathematics Subject Classification (2010): Primary 16S32, 17D25; Secondary 16R10, 17B01,17A50.

Key words: algebras of derivations, left-symmetric algebras, identities, free algebras, multiplication algebras.

\section{Introduction}

Let $k$ be an arbitrary field of characteristic zero and $P_{n}=k\left[x_{1}, x_{2}, \ldots, x_{n}\right]$ be the polynomial algebra over $k$ in the variables $x_{1}, x_{2}, \ldots, x_{n}$. Let $W_{n}$ be the Witt algebra of index $n$, i.e., Lie algebra of all derivations of the polynomial algebra $P_{n}$. The set of elements $u \partial_{i}$, where $u=x_{1}^{s_{1}} \ldots x_{n}^{s_{n}} \in P_{n}$ is an arbitrary monomial, $\partial_{i}=\frac{\partial}{\partial x_{i}}$, and $1 \leq i \leq n$, forms a linear basis for $W_{n}$. Denote by $\mathscr{L}_{n}$ an algebra with the ground space $W_{n}$ and the product $\cdot$ defined by

$$
a \partial_{i} \cdot b \partial_{j}=\left(a \partial_{i}(b)\right) \partial_{j}, \quad a, b \in P_{n} .
$$

It is easy to check [2] that $\mathscr{L}_{n}$ satisfies the left-symmetric identity

$$
(x y) z-x(y z)=(y x) z-y(x z) .
$$

This means that the associator $(x, y, z):=(x y) z-x(y z)$ is symmetric with respect to two left arguments, i.e.,

$$
(x, y, z)=(y, x, z) .
$$

The variety of left-symmetric algebras is Lie-admissible, i.e., each left-symmetric algebra $\mathscr{L}$ with the operation $[x, y]:=x y-y x$ is a Lie algebra. Obviously, the commutator algebra of $\mathscr{L}_{n}$ coincides with the Witt algebra $W_{n}$ of all derivations of $P_{n}$. The algebra $\mathscr{L}_{n}$ is called the left-symmetric Witt algebra of index $n$.

\footnotetext{
${ }^{1}$ Supported by an MES grant 0755/GF of Kazakhstan; Eurasian National University, Astana, Kazakhstan, e-mail: kozybayev@gmail.com

${ }^{2}$ Supported by an MES grant 0755/GF of Kazakhstan; Eurasian National University, Astana, Kazakhstan and Wayne State University, Detroit, MI 48202, USA, e-mail: umirbaev@math.wayne.edu
} 
The left-symmetric Witt algebra $\mathscr{L}_{n}$ of all derivations of the polynomial algebra $P_{n}$ plays an important role in the study of locally nilpotent derivations of $P_{n}$ and the Jacobian Conjecture [12. The set of all left nilpotent elements of $\mathscr{L}_{n}$ coincides with the set of all locally nilpotent derivations of the polynomial algebra $P_{n}$ and right multiplication operator $R_{D}$, where $D \in \mathscr{L}_{n}$, is nilpotent if and only if the Jacobian matrix of $D$ (see, definition in [12]) is nilpotent. These facts already lead to necessity of purely algebraic study of $\mathscr{L}_{n}$. First of all, it is important to get algebraic descriptions of left and right nilpotent elements of $\mathscr{L}_{n}$.

Identities of $\mathscr{L}_{n}$ are especially important in order to treat the mentioned above problems of affine algebraic geometry [12]. Notice that $\mathscr{L}_{1}$ is a Novikov algebra, i.e., it also satisfies the identity

$$
(x y) z=(x z) y \text {. }
$$

Moreover, the identities (2) and (3) form a basis of identities for $\mathscr{L}_{1}$ in characteristic zero [8]. Recall that the question on a basis of identities for $W_{1}$ is a well-known open problem [10]. A basis for Z-graded identities of the Lie algebra $W_{1}$ was recently given in [6].

The right multiplication algebra of $\mathscr{L}_{n}$, i.e., the associative algebra generated by all right multiplication operators $R_{x}, x \in \mathscr{L}_{n}$, is isomorphic to the matrix algebra $M_{n}\left(P_{n}\right)$ [12, Lemma 4]. This result allows to make some parallel between the matrix algebras $M_{n}(k)$ in the case of associative algebras and the left-symmetric Witt algebras $\mathscr{L}_{n}$ in the case of left-symmetric algebras. In Section 2, we describe all right operator identities of the left-symmetric Witt algebra $\mathscr{L}_{n}$ as well as its subalgebras of triangular derivations $T\left(\mathscr{L}_{n}\right)$ and strongly triangular derivations $S T\left(\mathscr{L}_{n}\right)$. Recall that minimal right operator identities of $\mathscr{L}_{n}$ are considered in [4].

These results give that

$$
\operatorname{Var}\left(\mathscr{L}_{1}\right) \varsubsetneqq \operatorname{Var}\left(\mathscr{L}_{2}\right) \varsubsetneqq \ldots \varsubsetneqq \operatorname{Var}\left(\mathscr{L}_{n}\right) \varsubsetneqq \ldots,
$$

where $\operatorname{Var}\left(\mathscr{L}_{n}\right)$ is the variety of algebras generated by $\mathscr{L}_{n}$.

In Section 4 , we prove that the set of all left-symmetric Witt algebras $\mathscr{L}_{n}$, where $n \geq 1$, generates the variety of all left symmetric algebras. This means that every polynomial identity satisfied by all algebras $\mathscr{L}_{n}$ is a consequence of (2). In fact, we prove more stronger result (Theorem 1), which says that the algebra of derivations $S T\left(\mathscr{L}_{n}\right)$ does not satisfy any nontrivial left-symmetric identity of degree $\leq n$.

Unfortunately, left operator identities of $\mathscr{L}_{n}$ are more important in applications and more difficult to describe. It is known that every Lie identity for $W_{n}$ is equivalent to a left operator identity for $\mathscr{L}_{n}$ [12, Lemma 7]. Identities of the Lie algebra $W_{n}$ are studied in [10]. In fact, Razmyslov's methods of proving identities for $W_{n}$ applied directly to $\mathscr{L}_{n}$ give more wider class of identities for $\mathscr{L}_{n}$. These identities are described in Section 5.

In Section 3, we give some auxiliary results on a basis of free left-symmetric algebras and some of them can be found in [7, 11].

\section{Algebras of triangular Derivations}


Every element $D$ of the left-symmetric Witt algebra $\mathscr{L}_{n}$ can be represented uniquely in the form

$$
D_{F}=f_{1} \partial_{1}+f_{2} \partial_{2}+\ldots+f_{n} \partial_{n},
$$

where $F=\left(f_{1}, f_{2}, \ldots, f_{n}\right)^{\prime}$ is an n-tuple (column) of elements of the polynomial algebra $P_{n}=k\left[x_{1}, x_{2}, \ldots, x_{n}\right]$. Let $J(F)=\left(\partial_{j}\left(f_{i}\right)\right)_{1 \leq i, j \leq n}$ be the Jacobian matrix of $F$ considered as a polynomial mapping of the space $k^{n}$. The Jacobian matrix $J(F)$ is also called the Jacobian matrix of the derivation $D_{F}$. Thereby, every derivation $D \in \mathscr{L}_{n}$ has the Jacobian matrix $J(D)=J\left(D_{F}\right)=J(F)$.

Consider the grading

$$
P_{n}=A_{0} \oplus A_{1} \oplus A_{2} \oplus \ldots \oplus A_{s} \oplus \ldots
$$

of the polynomial algebra $P_{n}$, where $A_{i}$ is the space of homogeneous elements of degree $i \geq 0$. The left-symmetric algebra $\mathscr{L}_{n}$ has a natural grading

$$
\mathscr{L}_{n}=L_{-1} \oplus L_{0} \oplus L_{1} \oplus \ldots \oplus L_{s} \oplus \ldots,
$$

where $L_{i}$ is the space generated by all elements of the form $a \partial_{j}$ with $a \in A_{i+1}$ and $1 \leq j \leq n$. Elements of $L_{s}$ are called homogeneous derivations of $P_{n}$ of degree $s$.

We have $L_{-1}=k \partial_{1}+\ldots+k \partial_{n}$ and $L_{0}$ is a subalgebra of $\mathscr{L}_{n}$ isomorphic to the matrix algebra $M_{n}(k)$. Notice that $L_{-1} \cdot L_{n} \subset L_{n-1}$ for all $n \geq 0$. If $0 \neq f \in L_{n}$ and $n \geq 0$, then, obviously, there exists $\partial_{s} \in L_{-1}$ such that $\partial_{s} \cdot f \neq 0$. Following [10], this property will be referred as the left transitivity of the grading (4).

The element

$$
D_{X}=x_{1} \partial_{1}+x_{2} \partial_{2}+\ldots+x_{n} \partial_{n}
$$

is the identity element of the matrix algebra $L_{0}$ and is the right identity element of $\mathscr{L}_{n}$. The left-symmetric algebra $\mathscr{L}_{n}$ has no identity element.

Let $A$ be an arbitrary left-symmetric algebra. Denote by $\operatorname{Hom}_{k}(A, A)$ the associative algebra of all $k$-linear transformations of the vector space $A$. For any $x \in A$ denote by $L_{x}: A \rightarrow A(a \mapsto x a)$ and $R_{x}: A \rightarrow A(a \mapsto a x)$ the operators of left and right multiplication by $x$, respectively. It follows from (2) that

$$
L_{[x, y]}=\left[L_{x}, L_{y}\right], \quad R_{x y}=R_{y} R_{x}+\left[L_{x}, R_{y}\right] .
$$

Denote by $M(A)$ the subalgebra of $\operatorname{Hom}_{k}(A, A)$ (with an identity) generated by all $R_{x}, L_{x}$, where $x \in A$. The algebra $M(A)$ is called the multiplication algebra of $A$. The subalgebra $R(A)$ of $M(A)$ (with identity) generated by all $R_{x}$, where $x \in A$, is called the right multiplication algebra of $A$. Similarly, the subalgebra $L(A)$ of $M(A)$ (with identity) generated by all $L_{x}$, where $x \in A$, is called the left multiplication algebra of $A$.

For shortness of terminology, we say that $f=f\left(z_{1}, \ldots, z_{m}\right)$ is an associative polynomial if $f$ is an element of the free associative algebra Ass $\left\langle z_{1}, \ldots, z_{m}\right\rangle$ freely generated by $z_{1}, \ldots, z_{m}$. If $f=f\left(z_{1}, \ldots, z_{m}\right) \in \operatorname{Ass}\left\langle z_{1}, \ldots, z_{m}\right\rangle$, then denote by $f^{*}$ the image of $f$ under the standard involution $*$ of $\operatorname{Ass}\left\langle z_{1}, \ldots, z_{m}\right\rangle$, i.e., $z_{i}^{*}=z_{i}$ for all $i$. We also say that $f=f\left(z_{1}, \ldots, z_{m}\right)$ is a left-symmetric polynomial if $f$ is an element of the free left-symmetric algebra $L S\left\langle z_{1}, \ldots, z_{m}\right\rangle$.

An identity $g=0$ of a left-symmetric algebra $A$ is called a right operator identity if $g=f\left(R_{x_{1}}, \ldots, R_{x_{m}}\right) x$ for some associative polynomial $f=f\left(z_{1}, \ldots, z_{m}\right)$. This means 
$f\left(R_{a_{1}}, \ldots, R_{a_{m}}\right)=0$ in $R(A)$ for all $a_{1}, \ldots, a_{m} \in A$. Notice that $f=0$ is not an identity for $R(A)$ (in general). Left operator identities can be defined similarly.

The right multiplication algebra $R\left(\mathscr{L}_{n}\right)$ of the left-symmetric Witt algebra $\mathscr{L}_{n}$ is isomorphic to the matrix algebra $M_{n}\left(P_{n}\right)$ [12]. This isomorphism

$$
\theta: R\left(\mathscr{L}_{n}\right) \longrightarrow M_{n}\left(P_{n}\right)
$$

is uniquely defined by $\theta\left(R_{D}\right)=J(D)$ for all $D \in \mathscr{L}_{n}$.

So the algebra $R\left(\mathscr{L}_{n}\right)$ satisfies all identities of the matrix algebra $M_{n}(k)$. Obviously, every identity of $R\left(\mathscr{L}_{n}\right)$ determines an identity (in fact, a set of identities) for $\mathscr{L}_{n}$. The isomorphism $\theta$ allows to carry out the study of $\mathscr{L}_{n}$ parallel to the study of $M_{n}(k)$. In this section we describe all right operator identities of $\mathscr{L}_{n}$ and its two important subalgebras.

The next proposition gives a complete description of all right operator identities of $\mathscr{L}_{n}$.

Proposition 1. Let $f=f\left(z_{1}, \ldots, z_{m}\right)$ be an associative polynomial. Then $f\left(z_{1}, \ldots, z_{m}\right)=$ 0 is an identity for $M_{n}(k)$ if and only if $f\left(R_{y_{1}}, \ldots, R_{y_{m}}\right) y=0$ is a right operator identity for $\mathscr{L}_{n}$.

Proof. Recall that $L_{0} \simeq M_{n}(k)$ is an associative algebra. We have

$$
\partial_{s} \cdot\left(x_{t} \partial_{r} \cdot x_{i} \partial_{j}\right)=\left(\partial_{s} \cdot x_{t} \partial_{r}\right) \cdot x_{i} \partial_{j}
$$

for all $s, t, r, i, j$. This means that $L_{-1}$ is a right $L_{0}$-module.

Transformation of matrices is an involution of $M_{n}(k)$. Consequently, $f=0$ is an identity for $M_{n}(k)$ if and only if $f^{*}=0$ is an identity for $M_{n}(k)$. Therefore, if $f=0$ is not an identity for $M_{n}(k)$, then there exist $a_{1}, \ldots, a_{m} \in L_{0}$ such that $0 \neq f^{*}\left(a_{1}, \ldots, a_{m}\right) \in L_{0}$. By the left transitivity of the grading (44), there exists $\partial \in L_{-1}$ such that $\partial\left(f^{*}\left(a_{1}, \ldots, a_{m}\right)\right) \neq$ 0 . Since $L_{-1}$ is a right $L_{0}$-module, we get

$$
\partial\left(f^{*}\left(a_{1}, \ldots, a_{m}\right)\right)=f\left(R_{a_{1}}, \ldots, R_{a_{m}}\right) \partial \neq 0 .
$$

If $f=0$ is an identity for $M_{n}(k)$, then $f=0$ is an identity for $R\left(\mathscr{L}_{n}\right)$ since $R\left(\mathscr{L}_{n}\right) \simeq$ $M_{n}\left(P_{n}\right)$. Consequently, $f\left(R_{a_{1}}, \ldots, R_{a_{m}}\right)=0$ for all $a_{1}, \ldots, a_{m} \in \mathscr{L}_{n}$. This means $f\left(R_{a_{1}}, \ldots, R_{a_{m}}\right) a=0$ for all $a \in \mathscr{L}_{n}$.

According to the Amitsur-Levitzki theorem [1], $M_{n}(k)$ satisfies the standard identity

$$
s_{2 n}\left(y_{1}, y_{2}, \ldots, y_{2 n}\right)=\sum_{\sigma \in S_{2 n}} \operatorname{sgn}(\sigma) y_{\sigma(1)} y_{\sigma(2)} \ldots y_{\sigma(2 n)}=0
$$

of degree $2 n$, where $S_{2 n}$ is the symmetric group on degree $2 n$ and $\operatorname{sgn}(\sigma)$ is the sign of the permutation $\sigma$. Moreover, $s_{2 n}$ is an identity of $M_{n}(k)$ of the minimal degree [1].

Corollary 1. [4] The left-symmetric Witt algebra $\mathscr{L}_{n}$ satisfies the identity

$$
s_{2 n}\left(R_{y_{1}}, R_{y_{2}}, \ldots, R_{y_{2 n}}\right) y_{2 n+1}=0
$$

of degree $2 n+1$ and this is a right operator identity of $\mathscr{L}_{n}$ of the minimal degree.

If $n=1$, this corollary gives the Novikov identity (3).

Let $T\left(\mathscr{L}_{n}\right)$ be the subspace of $\mathscr{L}_{n}$ generated by all derivations of the form

$$
x_{i}^{s_{i}} x_{i+1}^{s_{i+1}} \ldots x_{n}^{s_{n}} \partial_{i}
$$

where $s_{i}, s_{i+1}, \ldots, s_{n} \in \mathbb{Z}_{+}, \mathbb{Z}_{+}$is the set of all nonnegative integers, and $1 \leq i \leq n$. It is easy to check that $T\left(\mathscr{L}_{n}\right)$ is a subalgebra of $\mathscr{L}_{n}$. We call $T\left(\mathscr{L}_{n}\right)$ the algebra of all 
triangular derivations of $P_{n}$. In fact, $T\left(\mathscr{L}_{n}\right)$ is the set of all derivations $D$ such that $J(D)$ is an upper triangular matrix, i.e., $\theta\left(R_{D}\right)=J(D) \in T_{n}\left(P_{n}\right)$. Recall [9] that the identity

$$
\left[x_{1}, y_{1}\right]\left[x_{2}, y_{2}\right] \ldots\left[x_{n}, y_{n}\right]=0
$$

is a basis for all identities of the algebra $T_{n}(k)$ of all triangular matrices.

Corollary 2. Let $f=f\left(z_{1}, \ldots, z_{m}\right)$ be an arbitrary element of the free associative algebra $\operatorname{Ass}\left\langle z_{1}, \ldots, z_{m}\right\rangle$ and let $C$ be the commutator ideal of $\operatorname{Ass}\left\langle z_{1}, \ldots, z_{m}\right\rangle$. Then the following conditions are equivalent.

(i) $f\left(z_{1}, \ldots, z_{m}\right)=0$ is an identity for $T_{n}(k)$;

(ii) $f \in C^{n}$;

(iii) $f\left(R_{y_{1}}, \ldots, R_{y_{m}}\right) y=0$ is an identity for $T\left(\mathscr{L}_{n}\right)$.

Proof. Notice that (i) and (ii) are equivalent [9]. This gives that $f=0$ is an identity for $T_{n}(k)$ if and only if $f^{*}=0$ is an identity for $T_{n}(k)$. Notice that $L_{-1} \subset T\left(\mathscr{L}_{n}\right)$ and $T_{n}(k)=L_{0} \cap T\left(\mathscr{L}_{n}\right) \subset T\left(\mathscr{L}_{n}\right)$. Using these facts, we can repeat the proof of Proposition 1 for this case without any changes.

Denote by $S T\left(\mathscr{L}_{n}\right)$ the subspace of $\mathscr{L}_{n}$ generated by all derivations of the form

$$
x_{i+1}^{s_{i+1}} \ldots x_{n}^{s_{n}} \partial_{i},
$$

where $s_{i+1}, \ldots, s_{n} \in \mathbb{Z}_{+}$and $1 \leq i \leq n$. Then $S T\left(\mathscr{L}_{n}\right)$ is a subalgebra of $T\left(\mathscr{L}_{n}\right)$. We call $S T\left(\mathscr{L}_{n}\right)$ the algebra of all strongly triangular derivations of $P_{n}$. Notice that $S T\left(\mathscr{L}_{n}\right)$ is the set of all derivations $D$ such that $J(D)$ is an upper strongly triangular matrix, i.e., $J(D) \in S T_{n}\left(P_{n}\right)$. The algebra $S T_{n}(k)$ of all strongly triangular matrices satisfies the identity

$$
y_{1} y_{2} \ldots y_{n}=0 \text {. }
$$

It is easy to check that $S T_{n}(k)$ does not satisfy any nontrivial associative identity of degree less than $n$.

Repeating the proofs of Proposition 1 and Corollary 2, we get the next corollary.

Corollary 3. Every right operator identity of the algebra $S T\left(\mathscr{L}_{n}\right)$ of all strongly triangular derivations is a corollary of the identity

$$
R_{y_{1}} R_{y_{2}} \ldots R_{y_{n}} z=0
$$

So $S T\left(\mathscr{L}_{n}\right)$ is right nilpotent. It is not difficult to show that $S T\left(\mathscr{L}_{n}\right)$ is locally nilpotent but not nilpotent. In fact, the identity of right nilpotency does not give too much in the case of left-symmetric algebras. An example of a two-dimensional right nilpotent but not nilpotent Novikov algebra is given in [13]. More strange examples of Novikov algebras in small dimensions can be found in [3]. The picture might be totally different for subalgebras of $\mathscr{L}_{n}$.

Problem 1. Let $\mathscr{L}$ be a subalgebra of $\mathscr{L}_{n}$ satisfying the identity $R_{y}^{n} z=0$. Is $\mathscr{L}$ locally nilpotent?

Identities of the form $L_{y}^{m} z=0$ have stronger corollaries [5] in the case of left-symmetric algebras and related to the study of Engel Lie algebras [14]. 


\section{REDUCED WORDS}

Let $Y=\left\{y_{1}, y_{2}, \ldots, y_{n}\right\}$ be an alphabet of $n$ symbols. Denote by $Y^{*}$ the monoid of all nonassociative words on $Y$ [15]. If $u \in Y^{*}$, then denote by $d(u)$ the length of $u$. Every nonassociative word $u$ of length $\geq 2$ can be uniquely written as $u=u_{1} u_{2}$, where $d\left(u_{1}\right), d\left(u_{2}\right)<d(u)$. If $u \in Y^{*}$ and $d(u) \geq 2$, then we consider only nontrivial decompositions of $u$, i.e., if $u=u_{1} u_{2}$, then $d\left(u_{1}\right), d\left(u_{2}\right) \geq 1$.

For any $u \in Y^{*}$ denote by $[u]$ the associative word obtained from $u$ by skipping all parentheses.

Put $y_{1}<y_{2}<\ldots<y_{n}$. Let $u$ and $v$ be arbitrary elements of $Y^{*}$. We say that $u<v$ if $d(u)<d(v)$. If $d(u)=d(v) \geq 2, u=u_{1} u_{2}$, and $v=v_{1} v_{2}$, then $u<v$ if either $u_{1}<v_{1}$ or $u_{1}=v_{1}$ and $u_{2}<v_{2}$.

Put $\bar{n}=\{1,2, \ldots, n\}$. Every mapping $\sigma: \bar{n} \rightarrow \bar{n}$ will be identified with the endomorphism $\sigma: Y^{*} \rightarrow Y^{*}$ of the monoid $Y^{*}$ defined by $\sigma\left(y_{i}\right)=y_{\sigma(i)}$.

Lemma 1. Let $u, v \in Y^{*}$ be arbitrary two words such that $u>v,[u]=y_{i_{1}} y_{i_{2}} \ldots y_{i_{m}}$, $[v]=y_{j_{1}} y_{j_{2}} \ldots y_{j_{r}}$, and let $\sigma: \bar{n} \rightarrow \bar{n}$ be a mapping such that $\sigma\left(i_{1}\right)>\ldots>\sigma\left(i_{m}\right)>$ $\sigma\left(j_{1}\right)>\ldots>\sigma\left(j_{r}\right)$. Then $\sigma(u)>\sigma(v)$.

Proof. Assume that the statement of the proposition is not true and let $u, v \in Y^{*}$ be a counterexample with the minimal $d(u)+d(v)$. If $d(u)>d(v)$, then $\sigma(u)>\sigma(v)$ since $d(\sigma(u))>d(\sigma(v))$. Consequently, we may assume that $d(u)=d(v)$. If $d(u)=d(v)=1$ then $u=y_{i_{1}}, v=y_{j_{1}}$. Therefore, $\sigma(u)=y_{\sigma\left(i_{1}\right)}, \sigma(v)=y_{\sigma\left(j_{1}\right)}$, and $\sigma(u)>\sigma(v)$ since $\sigma\left(i_{1}\right)>\sigma\left(j_{1}\right)$.

If $d(u)=d(v) \geq 2$, then $u=u_{1} u_{2}$ and $v=v_{1} v_{2}$. If $u_{1}>v_{1}$, then $u_{1}, v_{1}$ satisfies all the conditions of the proposition and $d\left(u_{1}\right)+d\left(v_{1}\right)<d(u)+d(v)$. By the choice of $u, v$ we get $\sigma\left(u_{1}\right)>\sigma\left(v_{1}\right)$. Consequently, $\sigma(u)>\sigma(v)$. If $u_{1}=v_{1}$, then $u_{2}>v_{2}$. Similarly, we get $\sigma\left(u_{2}\right)>\sigma\left(v_{2}\right)$ and $\sigma(u)>\sigma(v)$.

A word $w \in Y^{*}$ is called reduced [11] if it does not contain any subword of the form $r(s t) \in Y^{*}$, where $d(r), d(s), d(t) \geq 1$ and $r<s$. Denote by $V$ the set of all reduced words in the alphabet $Y$.

Lemma 2. Let $w \in V,[w]=y_{i_{1}} y_{i_{2}} \ldots y_{i_{m}}$, and let $\sigma: \bar{n} \rightarrow \bar{n}$ be a mapping such that $\sigma\left(i_{1}\right)>\ldots>\sigma\left(i_{m}\right)$. Then $\sigma(w) \in V$.

Proof. If $d(w)=1$, then there is nothing to prove. Suppose that $d(w) \geq 2$ and the statement of the proposition is true for all reduced words of length less than $d(w)$. Let $w=u v$. Then $\sigma(u), \sigma(v) \in V$ by the induction proposition. If $d(v)=1$, then , obviously, $\sigma(w)=\sigma(u) \sigma(v) \in V$. If $v=v_{1} v_{2}$, then $\sigma(w)=\sigma(u)\left(\sigma\left(v_{1}\right) \sigma\left(v_{2}\right)\right)$. We have $u \geq v_{1}$ since $w \in V$. By Lemma 1, we get $\sigma(u) \geq \sigma\left(v_{1}\right)$. This implies that $\sigma(w) \in V$ since $\sigma(u), \sigma(v) \in V$.

Let $A=L S\langle Y\rangle$ be the free left-symmetric algebra with free set of generators $Y$. Every nonassociative word in the alphabet $Y$ represents a certain element of $A$ and for any $u \in Y^{*}$ we denote by $u$ the element of $A$ defined by $u$. 
According to [11], the set of all reduced words forms a linear basis for $A$ : every nonzero element $g$ of $A$ can be uniquely represented as

$$
g=\alpha_{1} w_{1}+\alpha_{2} w_{2}+\ldots+\alpha_{m} w_{m}
$$

where $w_{i} \in V, 0 \neq \alpha_{i} \in k$ for all $i$, and $w_{1}<w_{2}<\ldots<w_{m}$.

Denote by $\bar{g}$ the lowest word $w_{1}$ of $g$. The element $\alpha_{1} w_{1}$ is also called the lowest term of $g$.

Lemma 3. Let $w \in Y^{*}$ be an arbitrary nonassociative word. Then $\bar{w} \geq w$ in the algebra $A=L S\langle Y\rangle$ and the equality holds if and only if $w \in V$.

Proof. Assume that the statement of the lemma is true for all words of length less than $t$, where $t \geq 2$. Assume also that $w$ is the maximal word of length $t$ for which the statement of the lemma is not true. If $w \in V$, then $\bar{w}=w$. Hence $w=u v \notin V$. If $u$ or $v$ is not reduced, then, by the assumptions above, $w$ is a linear combination of words of the form $u^{\prime} v^{\prime}$ such that $u^{\prime} v^{\prime}>w$. By the choice of $w$, the statement of the lemma is true for all $u^{\prime} v^{\prime}$. Consequently, $w$ is a linear combination of reduced words $w^{\prime}$ such that $w^{\prime}>w$.

Suppose that both $u$ and $v$ are reduced. This means $v=v_{1} v_{2}$ and $u<v_{1}$ since $w$ is not reduced. By (2), we have

$$
w=u\left(v_{1} v_{2}\right)=v_{1}\left(u v_{2}\right)+\left(u v_{1}\right) v_{2}-\left(v_{1} u\right) v_{2}
$$

in the algebra $A$. Notice that $v_{1}\left(u v_{2}\right),\left(u v_{1}\right) v_{2},\left(v_{1} u\right) v_{2}>w$. By the choice of $w$, we again get that $w$ is a linear combination of reduced words $w^{\prime}$ such that $w^{\prime}>w$.

Lemma 4. Every reduced word $w \in V$ can be uniquely represented in the form

$$
w=L_{w_{1}} L_{w_{2}} \ldots L_{w_{m}} x_{i}
$$

where $w_{j} \in V$ for all $j$ and $w_{1} \geq w_{2} \geq \ldots \geq w_{m}$.

Proof. If $w=u v$, then $u$ and $v$ are reduced words. Leading an induction on $d(w)$, we may assume that $v=L_{v_{1}} L_{v_{2}} \ldots L_{v_{s}} x_{i}$, where $v_{j} \in V$ and $v_{1} \geq v_{2} \geq \ldots \geq v_{s}$. We have $v=v_{1} v^{\prime}$ where $v^{\prime}=L_{v_{2}} \ldots L_{v_{s}} x_{i}$. Note that $u \geq v_{1}$ since $w=u v=v\left(v_{1} v^{\prime}\right)$ is reduced. Consequently, $w=L_{u} L_{v_{1}} L_{v_{2}} \ldots L_{v_{s}} x_{i}$ and $u \geq v_{1} \geq v_{2} \geq \ldots \geq v_{s}$. The uniqueness of this representation is obvious.

\section{Generalized triangular Derivations}

Let $\lambda=\left(\lambda_{12}, \lambda_{13}, \ldots, \lambda_{1 n}, \lambda_{23}, \ldots, \lambda_{n-1 n}\right)$ be a tuple of independent commutative variables $\lambda_{i j}$, where $1 \leq i<j \leq n$. Denote by $k[\lambda]$ the polynomial algebra over $k$ in the variables $\lambda_{i j}$. Every element of $k[\lambda]$ can be written uniquely in the form

$$
\lambda^{s}=\lambda_{12}^{s 12} \lambda_{13}^{s 13} \ldots \lambda_{1 n}^{s_{1 n}} \lambda_{23}^{s_{23}} \ldots \lambda_{n-1 n}^{s_{n-1 n}},
$$

where $s=\left(s_{12}, s_{13}, \ldots, s_{1 n}, s_{23}, \ldots, s_{n-1 n}\right) \in \mathbb{Z}_{+}^{r}$ and $r=\frac{(n-1) n}{2}$. Denote by $\leq$ the lexicographic order on $\mathbb{Z}_{+}^{r}$. Put also $\lambda^{s}<\lambda^{t}$ if $s<t$ in $\mathbb{Z}_{+}^{r}$. If $0 \neq f \in k[\lambda]$ then denote by $\tilde{f}$ the leading monomial of $f$.

Denote by $S$ the set of formal symbols

$$
x_{1}^{f_{1}} \ldots x_{n}^{f_{n}}
$$


where $f_{1}, f_{2}, \ldots, f_{n} \in k[\lambda]$, and consider $S$ as a semigroup with respect to the product

$$
x_{1}^{f_{1}} \ldots x_{n}^{f_{n}} x_{1}^{g_{1}} \ldots x_{n}^{g_{n}}=x_{1}^{f_{1}+g_{1}} \ldots x_{n}^{f_{n}+g_{n}} .
$$

Notice that $S$ just is a multiplicative version of the additive semigroup $k[\lambda]^{n}$. Put also $\operatorname{deg}_{x_{i}}\left(x_{1}^{f_{1}} \ldots x_{n}^{f_{n}}\right)=f_{i}$ for all $i$.

Consider the set of formal symbols $u \partial_{i}$, where $u \in S$ and $1 \leq i \leq n$. Let $\mathscr{L}$ be the free $k[\lambda]$-module generated by all $u \partial_{i}$. We turn $\mathscr{L}$ into $k[\lambda]$-algebra by

$$
u \partial_{i} \circ v \partial_{j}=\operatorname{deg}_{x_{i}}(v)\left(u v x_{i}^{-1}\right) \partial_{j} .
$$

Lemma 5. $\mathscr{L}$ is a left-symmetric $k[\lambda]$-algebra.

Proof. If $u, v, w \in S$, then

$$
\begin{array}{r}
\left(u \partial_{i}, v \partial_{j}, w \partial_{k}\right)=\left(u \partial_{i} \circ v \partial_{j}\right) \circ w \partial_{k}-u \partial_{i} \circ\left(v \partial_{j} \circ w \partial_{k}\right) \\
=\operatorname{deg}_{x_{i}}(v)\left(u v x_{i}^{-1}\right) \partial_{j} \circ w \partial_{k}-\operatorname{deg}_{x_{j}}(w) u \partial_{i} \circ\left(v w x_{j}^{-1}\right) \partial_{k} \\
=\operatorname{deg}_{x_{i}}(v) \operatorname{deg}_{x_{j}}(w)\left(u v w x_{i}^{-1} x_{j}^{-1}\right) \partial_{k}-\operatorname{deg}_{x_{j}}(w) \operatorname{deg}_{x_{i}}\left(v w x_{j}^{-1}\right)\left(u v w x_{i}^{-1} x_{j}^{-1}\right) \partial_{k} \\
=\left(\operatorname{deg}_{x_{i}}(v) \operatorname{deg}_{x_{j}}(w)-\operatorname{deg}_{x_{j}}(w) \operatorname{deg}_{x_{i}}\left(v w x_{j}^{-1}\right)\right)\left(u v w x_{i}^{-1} x_{j}^{-1}\right) \partial_{k} \\
=-\operatorname{deg}_{x_{j}}(w)\left(\operatorname{deg}_{x_{i}}(w)+\operatorname{deg}_{x_{i}}\left(x_{j}^{-1}\right)\right)\left(u v w x_{i}^{-1} x_{j}^{-1}\right) \partial_{k} .
\end{array}
$$

Similarly, we get

$$
\left(v \partial_{j}, u \partial_{i}, w \partial_{k}\right)=-\operatorname{deg}_{x_{i}}(w)\left(\operatorname{deg}_{x_{j}}(w)+\operatorname{deg}_{x_{j}}\left(x_{i}^{-1}\right)\right)\left(u v w x_{i}^{-1} x_{j}^{-1}\right) \partial_{k} .
$$

If $i=j$, then these equalities give

$$
\left(u \partial_{i}, v \partial_{j}, w \partial_{k}\right)=\left(v \partial_{j}, u \partial_{i}, w \partial_{k}\right),
$$

i.e., the identity (2) holds for $u \partial_{i}, v \partial_{j}, w \partial_{k}$. If $i \neq j$, then $\operatorname{deg}_{x_{i}}\left(x_{j}^{-1}\right)=\operatorname{deg}_{x_{j}}\left(x_{i}^{-1}\right)=0$ and (2) holds again.

Denote by $\widehat{\mathscr{L}_{n}}$ the left-symmetric algebra of all derivations of $k\left[x_{1}^{ \pm 1}, x_{2}^{ \pm 1}, \ldots, x_{n}^{ \pm 1}\right]$. The set of elements $u \partial_{i}$, where $u=x_{1}^{s_{1}} \ldots x_{n}^{s_{n}}, s_{1}, \ldots, s_{n} \in \mathbb{Z}, \partial_{i}=\frac{\partial}{\partial x_{i}}$, and $1 \leq i \leq n$, forms a linear basis for $\widehat{\mathscr{L}}_{n}$. The product in $\widehat{\mathscr{L}}_{n}$ is defined by (1). The identity (2) can be checked as in the proof of Lemma 5 . The subalgebras of triangular derivations $T\left(\widehat{\mathscr{L}_{n}}\right)$ and strong triangular derivations $S T\left(\widehat{\mathscr{L}_{n}}\right)$ of $\widehat{\mathscr{L}_{n}}$ can be defined similarly.

For any $s=\left(s_{12}, s_{13}, \ldots, s_{1 n}, s_{23}, \ldots, s_{n-1 n}\right) \in \mathbb{Z}^{r}$ we define a $k$-linear mapping

$$
\widehat{s}: \mathscr{L} \longrightarrow \widehat{\mathscr{L}_{n}}
$$

by

$$
\widehat{s}\left(f(\lambda) x_{1}^{f_{1}(\lambda)} \ldots x_{n}^{f_{n}(\lambda)} \partial_{i}\right)=f(s) x_{1}^{f_{1}(s)} \ldots x_{n}^{f_{n}(s)} \partial_{i}
$$

for all $f, f_{1}, \ldots, f_{n} \in k[\lambda]$ and $1 \leq i \leq n$. Obviously, $\widehat{s}$ is defined correctly since $\mathscr{L}$ is a free $k[\lambda]$-module and $k[\lambda]$ is a free $k$-module.

Lemma 6. $\widehat{s}$ is a homomorphism of $k$-algebras. 
Proof. We have

$$
\begin{array}{r}
f(\lambda) x_{1}^{f_{1}(\lambda)} \ldots x_{n}^{f_{n}(\lambda)} \partial_{i} \circ g(\lambda) x_{1}^{g_{1}(\lambda)} \ldots x_{n}^{g_{n}(\lambda)} \partial_{j} \\
=f(\lambda) g(\lambda) g_{i}(\lambda) x_{1}^{f_{1}(\lambda)+g_{1}(\lambda)} \ldots x_{i}^{f_{i}(\lambda)+g_{i}(\lambda)-1} \ldots x_{n}^{f_{n}(\lambda)+g_{n}(\lambda)} \partial_{j}
\end{array}
$$

in $\mathscr{L}$. Obviously, we have

$$
\begin{array}{r}
f(s) x_{1}^{f_{1}(s)} \ldots x_{n}^{f_{n}(s)} \partial_{i} \cdot g(s) x_{1}^{g_{1}(s)} \ldots x_{n}^{g_{n}(s)} \partial_{j} \\
=f(s) g(s) g_{i}(s) x_{1}^{f_{1}(s)+g_{1}(s)} \ldots x_{i}^{f_{i}(s)+g_{i}(s)-1} \ldots x_{n}^{f_{n}(s)+g_{n}(s)} \partial_{j}
\end{array}
$$

in $\widehat{\mathscr{L}_{n}}$. This means that $\widehat{s}$ is a homomorphism of $k$-algebras.

Put

$$
u_{1}=x_{2}^{\lambda_{12}} \ldots x_{n}^{\lambda_{1 n}}, u_{2}=x_{3}^{\lambda_{23}} \ldots x_{n}^{\lambda_{2 n}}, \ldots, u_{n}=1 .
$$

Consider the elements

$$
z_{1}=u_{1} \partial_{1}, z_{2}=u_{2} \partial_{2}, \ldots, z_{n}=u_{n} \partial_{n}
$$

of $\mathscr{L}$ and denote by $\mathscr{L}(\lambda)$ the $k$-subalgebra of $\mathscr{L}$ generated by $z_{1}, z_{2}, \ldots, z_{n}$.

For any $s=\left(s_{12}, s_{13}, \ldots, s_{1 n}, s_{23}, \ldots, s_{n-1 n}\right) \in \mathbb{Z}_{+}^{r}$, consider the homomorphism $\widehat{s}$ defined by (8). Denote the restriction of $\widehat{s}$ into $\mathscr{L}(\lambda)$ by the same symbol $\widehat{s}$. Notice that $\widehat{s}(\mathscr{L}(\lambda)) \subset S T\left(\mathscr{L}_{n}\right)$ since $\widehat{s}\left(z_{i}\right) \in S T\left(\mathscr{L}_{n}\right)$. Finally, we have a homomorphism

$$
\widehat{s}: \mathscr{L}(\lambda) \longrightarrow S T\left(\mathscr{L}_{n}\right) \text {. }
$$

As in Section 3, let $A=L S\langle Y\rangle$ be the free left-symmetric algebra generated by $Y=$ $\left\{y_{1}, y_{2}, \ldots, y_{n}\right\}$. Denote by

$$
\chi: A \longrightarrow \mathscr{L}(\lambda)
$$

the homomorphism of $k$-algebras such that $\chi\left(y_{i}\right)=z_{i}$ for all $i$.

Lemma 7. Let $w \in Y^{*}$. Then there exist unique polynomials $f^{w}, f_{1}^{w}, \ldots, f_{n}^{w} \in k[\lambda]$ and a number $r(w)(1 \leq r(w) \leq n)$ such that

$$
\chi(w)=f^{w} x_{1}^{f_{1}^{w}} \ldots x_{n}^{f^{w}} \partial_{r(w)} .
$$

Proof. If $w=y_{i}$ then $\chi(w)=z_{i}$. Hence $f^{w}=1, f_{1}^{w}=\ldots=f_{i}^{w}=0, f_{i+1}^{w}=$ $\lambda_{i i+1}, \ldots, f_{n}^{w}=\lambda_{i n}$, and $r(w)=i$. If $w=u v$, then

$$
\begin{array}{r}
\chi(w)=\chi(u) \circ \chi(w)=f^{u} x_{1}^{f_{1}^{u}} \ldots x_{n}^{f_{n}^{u}} \partial_{r(u)} \circ f^{v} x_{1}^{f_{1}^{v}} \ldots x_{n}^{f_{n}^{v}} \partial_{r(v)} \\
=f^{u} f^{v} f_{r(u)}^{v} x_{1}^{f_{1}^{u}+f_{1}^{v}} \ldots x_{r(u)}^{f_{r(u)}^{u}+f_{r(u)}^{v}-1} \ldots x_{n}^{f_{n}^{u}+f_{n}^{v}} \partial_{r(v)}
\end{array}
$$

by the definition of the product $\circ$ since $\operatorname{deg}_{x_{r(u)}}\left(x_{1}^{f_{1}^{v}} \ldots x_{n}^{f_{n}^{v}}\right)=f_{r(u)}^{v}$.

We use this lemma also as the definition of $f^{w}, f_{i}^{w}$, and $r(w)$. The proof of this lemma also implies the next corollaries.

Corollary 4. Let $w \in Y^{*}$ and $w=u v$. Then $f^{w}=f^{u} f^{v} f_{r(u)}^{v}, f_{i}^{w}=f_{i}^{u}+f_{i}^{v}$ if $i \neq r(u)$, $f_{r(u)}^{w}=f_{r(u)}^{u}+f_{r(u)}^{v}-1$, and $r(w)=r(v)$.

Corollary 5. Let $w \in Y^{*}$ and $[w]=y_{i_{1}} \ldots y_{i_{m}}$. Then $r(w)=i_{m}$ and

$$
\chi(w)=f^{w} u_{i_{1}} u_{i_{2}} \ldots u_{i_{m}} x_{i_{1}}^{-1} x_{i_{2}}^{-1} \ldots x_{i_{m-1}}^{-1} \partial_{i_{m}} .
$$


Proof. Formula $r(w)=r(v)$ from Corollary 4 immediately gives $r(w)=i_{m}$.

If $d(w)=1$ and $w=y_{i_{1}}$, then $\chi(w)=z_{i_{1}}=u_{i_{1}} \partial_{i_{1}}$. Let $w=u v,[u]=y_{i_{1}} \ldots y_{i_{m}}$, and $[v]=y_{j_{1}} \ldots y_{j_{r}}$. Leading an induction on $d(w)$, we get

$$
\begin{array}{r}
\chi(w)=\chi(u) \circ \chi(v)=f^{u} u_{i_{1}} \ldots u_{i_{m}} x_{i_{1}}^{-1} \ldots x_{i_{m-1}}^{-1} \partial_{i_{m}} \circ f^{v} u_{j_{1}} \ldots u_{j_{r}} x_{j_{1}}^{-1} \ldots x_{j_{r-1}}^{-1} \partial_{j_{r}} \\
=f^{w} u_{i_{1}} \ldots u_{i_{m}} u_{j_{1}} \ldots u_{j_{r}} x_{i_{1}}^{-1} \ldots x_{i_{m-1}}^{-1} x_{j_{1}}^{-1} \ldots x_{j_{r-1}}^{-1} x_{i_{m}}^{-1} \partial_{j_{r}}
\end{array}
$$

since $f^{w}=f^{u} f^{v} f_{i_{m}}^{v}=f^{u} f^{v} \operatorname{deg}_{x_{i_{m}}}\left(u_{j_{1}} \ldots u_{j_{r}} x_{j_{1}}^{-1} \ldots x_{j_{r-1}}^{-1}\right)$. This implies the statement of the corollary.

By this corollary, $r(w)$ is the index of the rightmost symbol of $w$. We use this fact as a denotation.

Recall that a word $w \in Y^{*}$ is called multilinear if $d_{y_{i}}(w) \leq 1$ for all $i$, where $d_{y_{i}}(w)$ is the length of $w$ in $y_{i}$.

A word $w \in Y^{*}$ with $[w]=y_{i_{1}} y_{i_{2}} \ldots y_{i_{m}}$ is called an $s$-word if $i_{1}, i_{2}, \ldots, i_{m-1}>i_{m}$. We say $w \in Y^{*}$ is special if every subword of $w$ is an $s$-word.

Notice that if $w$ is a reduced word, $[w]=y_{i_{1}} y_{i_{2}} \ldots y_{i_{m}}$, and $i_{1}>i_{2}>\ldots>i_{m}$, then $w$ is a special reduced word. Obviously, every special reduced word $w$ has a unique representation in the form (77), where $w_{1}, w_{2}, \ldots, w_{m}$ are special reduced words.

Lemma 8. Let $v \in Y^{*}$ be a multilinear word. If $v$ is not special, then $\chi(v)=0$

Proof. Let $u$ be a nonspecial subword of $v$ of the minimal length. Then $u$ is not an $s$-word. Let $[u]=y_{i_{1}} \ldots y_{i_{m}}$ and let $i_{j}$ be the smallest number between $i_{1}, i_{2}, \ldots, i_{m}$. Notice that all numbers $i_{1}, i_{2}, \ldots, i_{m}$ are different since $v$ is multilinear and $j<m$ since $u$ is not an $s$-word. Consequently, $i_{j+1}, i_{j+2}, \ldots, i_{m}>i_{j}$. This means that $\chi\left(y_{i_{j+1}}\right)=$ $z_{i_{j+1}}, \chi\left(y_{i_{j+2}}\right)=z_{i_{j+2}}, \ldots, \chi\left(y_{i_{m}}\right)=z_{i_{m}}$ do not depend on $x_{i_{j}}$. Consequently, $\chi\left(y_{i_{j}}\right)=z_{i_{j}}$ annihilates the product $\chi\left(y_{i_{1}}\right) \ldots \chi\left(y_{i_{m}}\right)$ for any placement of parentheses.

Denote by $W$ the set of all multilinear special reduced words in the alphabet $Y$. If $w \in W$ and $[u]=y_{i_{1}} \ldots y_{i_{m}}$, then put $s(w)=\left\{i_{1}, i_{2}, \ldots, i_{m}\right\}$.

Lemma 9. Let $w \in W$ and $w=u v$. Then

$$
f_{r(u)}^{v}=\sum_{i \in s(v)} \lambda_{i r(u)} .
$$

Proof. Recall that $\lambda_{i j}=0$ if $i \geq j$. Consequently, only the indexes $i \in s(v)$ with $i<r(v)$ contribute to the sum in the formulation of the lemma.

Notice that $v$ does not contain $y_{r(u)}$ since $w$ is multilinear and $y_{r(u)}$ is the rightmost symbol of $u$. Consequently, we do not have $z_{r(u)}$ in $\chi(v)$. This means that the degrees in $x_{r(u)}$ of all elements participating in the product $\chi(v)$ will be summarized. Notice that the degree $x_{r(u)}$ in $z_{i}$ is $\lambda_{i r(u)}$ if $i<r(u)$ and 0 otherwise. This gives the statement of the lemma.

Lemma 10. Let $w \in W$ represented in the form (7). Then

$$
\widetilde{f^{w}}=\lambda_{i r\left(w_{1}\right)} \lambda_{i r\left(w_{2}\right)} \ldots \lambda_{i r\left(w_{m}\right)} \widetilde{f^{w_{1}}} \widetilde{f^{w_{2}}} \ldots \widetilde{f^{w_{m}}} .
$$


Proof. We have $w=w_{1} u$, where $u=L_{w_{2}} \ldots L_{w_{m}} y_{i}$. By Corollary 4, $f^{w}=f^{w_{1}} f^{u} f_{r\left(w_{1}\right)}^{u}$. By Lemma 9, we get

$$
f_{r\left(w_{1}\right)}^{u}=\sum_{j \in s(u)} \lambda_{j r\left(w_{1}\right)} .
$$

Notice that $i$ is the least number of $s(u)$ and $s(w)$ since $u$ and $w$ are special. Consequently, $\widetilde{f_{r\left(w_{1}\right)}^{u}}=\lambda_{i r\left(w_{1}\right)}$ and

$$
\widetilde{f^{w}}=\lambda_{i r\left(w_{1}\right)} \widetilde{f^{w_{1}}} \widetilde{f^{u}} .
$$

Leading an induction on $d(w)$, we may assume that

$$
\widetilde{f^{u}}=\lambda_{i r\left(w_{2}\right)} \ldots \lambda_{i r\left(w_{m}\right)} \widetilde{f^{w_{2}}} \ldots \widetilde{f^{w_{m}}} .
$$

This completes the proof of the lemma.

Corollary 6. In the conditions of Lemma 10 the following statements are true:

(i) $\widetilde{f^{w}}$ is a product of $d(w)-1$ elements $\lambda_{s t}$;

(ii) The least first index of all divisors $\lambda_{s t}$ of $\widetilde{f^{w}}$ is $i$;

(iii) The set of all second indexes of all divisors $\lambda_{s t}$ of $\widetilde{f^{w}}$ is equal to $s(w) \backslash\{i\}$;

(iv) Let $\lambda_{i j_{1}}, \lambda_{i j_{2}}, \ldots, \lambda_{i j_{p}}$ be the set of all divisors $\lambda_{s t}$ of $\widetilde{f^{w}}$ with the first index $i$. Then $p=m$ and $\left\{j_{1}, j_{2}, \ldots, j_{p}\right\}=\left\{r\left(w_{1}\right), r\left(w_{2}\right), \ldots, r\left(w_{m}\right)\right\}$.

Proof. Every statement of the corollary is obvious or can be deduced from Lemma 10 by an easy induction on $d(w)$.

Lemma 11. Every $w \in W$ is uniquely defined by $\widetilde{f^{w}}$.

Proof. We determine $w \in W$ in the form (77). By Corollary 6(ii), $r(w)=i$ is determined uniquely. By Corollary 6 (iv), the number $m$ and the set of elements $\left\{r\left(w_{1}\right), r\left(w_{2}\right), \ldots, r\left(w_{m}\right)\right\}$ are also determined uniquely.

We introduce new orders $\succ$ and $\vdash$ on $s(w)$. If $p, q \in s(w)$, then put $q \succ p$ if $\lambda_{p q}$ divides $\widetilde{f^{w}}$. Put also $q \vdash p$ if $q=q_{0} \succ q_{1} \succ \ldots \succ q_{t}=p$ for some $t \geq 1$. By Corollary [6(iii), for any $q \in s(w) \backslash\{i\}$ there exists a unique element $p \in s(w)$ such that $q \succ p$. Lemma 10 implies that $q \vdash r\left(w_{j}\right)$ if and only if $q \in s\left(w_{j}\right) \backslash\left\{r\left(w_{j}\right)\right\}$. This fact uniquely determines $s\left(w_{j}\right)$ for all $j$. Notice that $\widetilde{f^{w_{j}}}$ is the product of all divisors $\lambda_{s t}$ of $\widetilde{f^{w}}$ such that $t \vdash r\left(w_{j}\right)$. Leading an induction on $d(w)$, we may assume that $w_{j}$ is uniquely determined by $\frac{f^{w_{j}}}{\text {. }}$. Then $w$ is also determined uniquely since $w_{1} \geq w_{2} \geq \ldots \geq w_{m}$.

Theorem 1. The left-symmetric algebra of all strongly triangular derivations $S T\left(\mathscr{L}_{n}\right)$ does not satisfy any nontrivial left-symmetric identity of degree less than or equal to $n$.

Proof. Recall [15] that every polynomial identity over a field of characteristic zero is equivalent to homogeneous multilinear polynomial identities. Since $S T\left(\mathscr{L}_{n-1}\right) \subset S T\left(\mathscr{L}_{n}\right)$, it is sufficient to prove that every multilinear polynomial identity of $S T\left(\mathscr{L}_{n}\right)$ of degree $n$ is a corollary of (2). Suppose that it is not true. Then there exists a nonzero multilinear element $g=g\left(y_{1}, y_{2}, \ldots, y_{n}\right)$ of the free left-symmetric algebra $A=L S\langle Y\rangle$ which is a polynomial identity for $S T\left(\mathscr{L}_{n}\right)$, i.e.,

$$
g\left(a_{1}, a_{2}, \ldots, a_{n}\right)=0
$$


for all $a_{1}, a_{2}, \ldots, a_{n} \in \mathscr{L}_{n}$. We fix this element $g$ and assume that $g$ is written in the form (6). Then $\bar{g}=w_{1}$

Let $[\bar{g}]=\left[w_{1}\right]=y_{i_{1}} y_{i_{2}} \ldots y_{i_{n}}$. We have $\left\{i_{1}, i_{2}, \ldots, i_{n}\right\}=\{1,2, \ldots, n\}$ since $g$ is multilinear of degree $n$. Consider the mapping $\sigma: \bar{n} \rightarrow \bar{n}$ defined $\sigma\left(i_{j}\right)=n-j+1$ for all $j$. Notice that $\sigma\left(i_{1}\right)>\sigma\left(i_{2}\right)>\ldots>\sigma\left(i_{n}\right)$. Denote by $\sigma$ the automorphism of the monoid $Y^{*}$ and the automorphism of the free algebra $A$ defined by $\sigma$. We have

$$
\sigma(g)=\alpha_{1} \sigma\left(w_{1}\right)+\alpha_{2} \sigma\left(w_{2}\right)+\ldots+\alpha_{m} \sigma\left(w_{m}\right) .
$$

By Lemma 1, we get $\sigma\left(w_{1}\right)<\sigma\left(w_{2}\right)<\ldots<\sigma\left(w_{m}\right)$. By Lemma 2, $\sigma\left(w_{1}\right)$ is a reduced word. Then Lemma 3 gives that $\overline{\sigma(g)}=\sigma\left(w_{1}\right)$.

Obviously, $\sigma\left(w_{1}\right)$ is special since $\left[\sigma\left(w_{1}\right)\right]=y_{n} y_{n-1} \ldots y_{1}$.

Consequently, by changing $g$ to $\sigma(g)$, we may assume that $\bar{g}=w_{1} \in W$. Let $g=h+h_{1}$, where $h$ is a linear combination of special reduced words and $h_{1}$ is a linear combination of reduced nonspecial words. We have $h \neq 0$ since $\bar{g} \in W$. We also have $\chi\left(h_{1}\right)=0$ by Lemma 8. Assume that

$$
h=\beta_{1} v_{1}+\beta_{2} v_{2}+\ldots+\beta_{s} v_{s},
$$

where $v_{i} \in W, 0 \neq \beta_{i} \in k, s \geq 1$, and $1 \leq i \leq s$. Notice that $r\left(v_{i}\right)=1$ for all $i$ since $v_{i}$ is special. By Corollary 5, we get $\chi\left(v_{i}\right)=f^{v_{i}} u_{1} u_{2} \ldots u_{n}\left(x_{2} x_{3} \ldots x_{n}\right)^{-1} \partial_{1}$ for all $i$. Hence

$$
\chi(g)=\chi(h)=\left(\beta_{1} f_{v_{1}}+\beta_{2} f_{v_{2}}+\ldots+\beta_{s} f_{v_{s}}\right) u_{1} u_{2} \ldots u_{n}\left(x_{2} x_{3} \ldots x_{n}\right)^{-1} \partial_{1} .
$$

Put $f_{g}=\beta_{1} f_{v_{1}}+\beta_{2} f_{v_{2}}+\ldots+\beta_{s} f_{v_{s}}$. By Lemma 9, the elements $\widetilde{f_{v_{i}}}$ are different for different values of $i$. Consequently, $f_{g} \in k[\lambda]$ is nonzero. It is not difficult to show that there exists $s \in \mathbb{Z}_{+}^{r}$ such that $f_{g}(s) \neq 0$. Then the image of $g$ under the homomorphism

$$
\widehat{s} \circ \chi: A \longrightarrow S T\left(\mathscr{L}_{n}\right)
$$

is nonzero. Consequently, $g$ is not an identity for $S T\left(\mathscr{L}_{n}\right)$.

Corollary 7. The identity (5) is a left-symmetric polynomial identity of $S T\left(\mathscr{L}_{n}\right)$ of the minimal degree.

Corollary 8. The variety of algebras generated by all algebras of strongly triangular derivations $S T\left(\mathscr{L}_{n}\right)$, where $n \geq 1$, is the variety of all left-symmetric algebras.

Corollary 9. The variety of algebras generated by all algebras of triangular derivations $T\left(\mathscr{L}_{n}\right)$, where $n \geq 1$, is the variety of all left-symmetric algebras.

Corollary 10. The variety of algebras generated by all left-symmetric Witt algebras $\mathscr{L}_{n}$, where $n \geq 1$, is the variety of all left-symmetric algebras.

\section{General identities}

A very interesting class of identities for $W_{n}$ was discovered by Yu.P. Razmyslov [10, Chapter 6]. Of course, every identity for $W_{n}$ is an identity for $\mathscr{L}_{n}$. Moreover, every identity for $W_{n}$ also gives a left operator identity for $\mathscr{L}_{n}$ [12, Lemma 7]. But Razmyslov's method, applied directly to $\mathscr{L}_{n}$, gives more wider class of identities for $\mathscr{L}_{n}$. We describe this class of identities. 
Consider the grading (4) of $\mathscr{L}_{n}$. Recall that $0 \neq a \in L_{i}$ is called homogeneous of degree $i$. Put $|a|=i$ in this case. Choose a homogeneous basis

$$
e_{1}, e_{2}, \ldots, e_{s}, \ldots
$$

for $\mathscr{L}_{n}$ such that $\left|e_{i}\right| \leq\left|e_{j}\right|$ if $i<j$. Then $e_{1}, \ldots, e_{n}$ is a basis for $L_{-1}$ and $e_{n+1}, \ldots, e_{n^{2}+n}$ is a basis for $L_{0}$, and so on.

For any positive integer $N$ put

$$
e(N)=\sum_{i=1}^{N}\left|e_{i}\right|
$$

Recall that a (nonassociative) polynomial $f=f\left(y_{1}, \ldots, y_{N}, z_{1}, \ldots, z_{t}\right)$ is called skewsymmetric with respect to $y_{1}, \ldots, y_{N}$ if

$$
\sigma(f)=f\left(y_{\sigma(1)}, \ldots, y_{\sigma(N)}, z_{1}, \ldots, z_{t}\right)=\operatorname{sgn}(\sigma) f\left(y_{1}, \ldots, y_{N}, z_{1}, \ldots, z_{t}\right)
$$

for all $\sigma \in S_{N}$, where $S_{N}$ is the group of degree $N$.

Proposition 2. Let $N$ be an arbitrary positive integer and $f=f\left(y_{1}, \ldots, y_{N}, z_{1}, \ldots, z_{t}\right)$ be an arbitrary multilinear left-symmetric polynomial skew-symmetric with respect to $y_{1}, \ldots, y_{N}$. If $e(N) \geq t$, then $f=0$ is an identity for $\mathscr{L}_{n}$.

Proof. The identity (2) gives

$$
\partial \cdot(u \cdot v)=(\partial \cdot u) \cdot v+u \cdot(\partial \cdot v)
$$

for all $\partial \in L_{-1}$ and $u, v \in \mathscr{L}_{n}$ since $\mathscr{L}_{n} \cdot L_{-1}=0$. This means that $L_{\partial}$ is a derivation of $\mathscr{L}_{n}$.

Let $g=g\left(z_{1}, \ldots, z_{l}\right)$ be an arbitrary multilinear left-symmetric polynomial. If $g=0$ is not an identity for $\mathscr{L}_{n}$, then there exist homogeneous elements $v_{1}, \ldots, v_{l} \in \mathscr{L}_{n}$ such that $0 \neq g\left(v_{1}, \ldots, v_{l}\right) \in L_{-1}$. In fact, let $v_{1}, \ldots, v_{l} \in \mathscr{L}_{n}$ be homogeneous elements such that $0 \neq g\left(v_{1}, \ldots, v_{l}\right) \in L_{i}$ and $i \geq 0$. By the left transitivity of the grading (4), there exists $\partial \in L_{-1}$ such that $L_{\partial} g\left(v_{1}, \ldots, v_{l}\right) \neq 0$. We have

$$
L_{\partial} g\left(v_{1}, \ldots, v_{l}\right)=\sum_{i=1}^{l} g\left(v_{1}, \ldots, L_{\partial} v_{i}, \ldots, v_{l}\right) .
$$

since $L_{\partial}$ is a derivation. Then there exists $i$ such that $0 \neq g\left(v_{1}, \ldots, L_{\partial} u_{i}, \ldots, v_{l}\right) \in L_{i-1}$.

Suppose that $f=0$ is not an identity for $\mathscr{L}_{n}$. Then there exist basis elements $e_{i_{1}}, \ldots, e_{i_{N}}, e_{j_{1}}, \ldots, e_{j_{t}} \in \mathscr{L}_{n}$ such that $0 \neq f\left(e_{i_{1}}, \ldots, e_{i_{N}}, e_{j_{1}}, \ldots, e_{j_{t}}\right) \in L_{-1}$. If two of the elements $e_{i_{1}}, \ldots, e_{i_{N}}$ are equal to each other, then $f\left(e_{i_{1}}, \ldots, e_{i_{N}}, e_{j_{1}}, \ldots, e_{j_{t}}\right)=0$ since $f$ is skew-symmetric with respect to $y_{1}, \ldots, y_{N}$. If all $e_{i_{1}}, \ldots, e_{i_{N}}$ are different, then

$$
\left|e_{i_{1}}\right|+\ldots+\left|e_{i_{N}}\right| \geq\left|e_{1}\right|+\ldots+\left|e_{N}\right|=e(N) .
$$

This implies that $f\left(e_{i_{1}}, \ldots, e_{i_{N}}, e_{j_{1}}, \ldots, e_{j_{t}}\right) \in L_{s}$ and $s \geq e(N)-t \geq 0$.

Let's describe the identities of the minimal degree given by Proposition 2 . Notice that $\left|e_{1}\right|+\ldots+\left|e_{n}\right|=-n$ and $\left|e_{n+1}\right|+\ldots+\left|e_{n^{2}+n}\right|=0$. The minimal $N$ for which $e(N)$ is 
nonnegative is $n^{2}+2 n$ and $e\left(n^{2}+2 n\right)=0$. Put $N=n^{2}+n$. Let $w$ be an arbitrary reduced word such that $[w]=y_{1} \ldots y_{N}$. Then

$$
S_{N}^{w}=\sum_{\sigma \in S_{N}} \operatorname{sgn}(\sigma) w\left(y_{\sigma(1)}, \ldots, y_{\sigma(N)}\right)=0
$$

is an identity for $\mathscr{L}_{n}$.

\section{REFERENCES}

[1] S.A. Amitsur and J. Levitzki, Minimal identities for algebras, Proc. Amer. Math. Soc. 1 (1950), 449-463.

[2] D. Burde, Left-symmetric algebras, or pre-Lie algebras in geometry and physics, Cent. Eur. J. Math. 4 (2006), no. 3, 323-357.

[3] D. Burde, W. de Graaf, Classification of Novikov algebras, Appl. Algebra Engrg. Comm. Comput. 24 (2013), no. 1, 1-15.

[4] A. Dzhumadil'daev, Minimal identities for right-symmetric algebras, J. Algebra 225 (2000), no. 1, 201-230.

[5] V.T. Filippov, On right-symmetric and Novikov nil algebras of bounded index, (Russian) Mat. Zametki 70 (2001), no. 2, 289-295; translation in Math. Notes 70 (2001), no. 1-2, 258-263.

[6] J.A. Freitas, P. Koshlukov, A. Krasilnikov, Z-graded identities of the Lie algebra $W_{1}$, J. Algebra 427 (2015), 226-251.

[7] D. Kozybaev, L. Makar-Limanov, U. Umirbaev, The Freiheitssatz and the automorphisms of free right-symmetric algebras, Asian-European Journal of Mathematics, Vol. 1, No. 2 (2008), 243-254.

[8] L. Makar-Limanov, U. Umirbaev, The Freiheitssatz for Novikov algebras, TWMS Jour. Pure Appl. Math., 2 (2011), no. 2, 66-73.

[9] Yu. Maltsev, A basis for the identities of the algebra of upper triangular matrices, (Russian) Algebra i Logika 10 (1971), 393-400.

[10] Yu.P. Razmyslov, Identities of algebras and their representations, Translated from the 1989 Russian original by A. M. Shtern. Translations of Mathematical Monographs, 138. American Mathematical Society, Providence, RI, 1994.

[11] D. Segal, Free left-symmetric algebras and an analogue of the Poincare-Birkhoff-Witt Theorem, J. Algebra 164 (1994), 750-752.

[12] U.U. Umirbaev, Associative, Lie, and left-symmetric algebras of derivations, Transformation Groups, 17 pages (accepted) (arXiv:1412.2840 [math.AG]).

[13] E.I. Zelmanov, A class of local translation-invariant Lie algebras, (Russian) Dokl. Akad. Nauk SSSR 292 (1987), no. 6, 1294-1297.

[14] E.I. Zelmanov, On Engel Lie algebras, (Russian) Sibirsk. Mat. Zh. 29 (1988), no. 5, 112-117; English translation: In Siberian Math. J. 29 (1988), no. 5, 777-781.

[15] K.A. Zhevlakov, A.M. Slinko, I.P. Shestakov, A.I. Shirshov, Rings that are nearly associative, Academic Press, Inc. [Harcourt Brace Jovanovich, Publishers], New York-London, 1982. 